\title{
Lire le texte polémique : violence du discours et plaisir de la lecture
}

Pascale Roux

\section{OpenEdition}

1 Journals

Édition électronique

URL : http://journals.openedition.org/recherchestravaux/662

DOI : 10.4000/recherchestravaux.662

ISSN : 1969-6434

Éditeur

UGA Éditions/Université Grenoble Alpes

Édition imprimée

Date de publication : 31 décembre 2013

Pagination : 25-39

ISBN : 978-2-84310-267-7

ISSN : 0151-1874

Référence électronique

Pascale Roux, "Lire le texte polémique : violence du discours et plaisir de la lecture », Recherches \& Travaux [En ligne], 83 | 2013, mis en ligne le 01 juillet 2015, consulté le 08 septembre 2020. URL:

http://journals.openedition.org/recherchestravaux/662; DOI : https://doi.org/10.4000/ recherchestravaux.662 


\section{Lire le texte polémique : violence du discours et plaisir de la lecture}

Les textes polémiques sont assez peu représentés dans le corpus des «classiques» étudiés dans l'institution scolaire et même universitaire. On peut s'étonner de cette exclusion, qui s'explique sans doute par plusieurs raisons. D'abord se pose la question de la littérarité du texte polémique, qui vise avant tout à être efficace, mais peut tout à fait mettre les ressources de la langue littéraire au service de l'efficacité du discours. Deuxième raison : les textes polémiques qui, par définition, sont des écrits de circonstance, exigent une connaissance du contexte dans lequel ils ont été publiés, ce qui complique peut-être leur utilisation dans le cadre d'un cours. Pour résoudre ce problème, il est possible de sélectionner des textes pour lesquels le contexte est moins lourd à exposer. On peut par ailleurs admettre la validité d'une lecture "actualisante ${ }^{\mathrm{I}}$ », qui génère certes une déformation ou un déplacement des enjeux d'origine, mais qui, si l'objectif n'est pas d'histoire littéraire, n'empêche pas nécessairement de faire apparaître les mécanismes textuels. En fait, le véritable problème que pose le texte polémique, celui qui justifie peut-être son exclusion du corpus scolaire et universitaire, est lié à la place du lecteur face à ce type de discours. Le lecteur

I. Y. Citton, Lire, interpréter, actualiser, Paris, Éditions Amsterdam, 2007. Y. Citton définit ainsi la notion que nous lui empruntons : "Une interprétation littéraire d'un texte ancien est actualisante dès lors que a) elle s'attache à exploiter les virtualités connotatives des signes de ce texte, b) afin d'en tirer une modélisation capable de reconfigurer un problème propre à la situation historique de l'interprète, c) sans viser à correspondre à la réalité historique de l'auteur, mais d) en exploitant, lorsque cela est possible, la différence entre les deux époques (leur langue, leur outillage mental, leurs situations socio-politiques) pour apporter un éclairage dépaysant sur le présent.» 
y est en effet le témoin d'une violence symbolique qui rend problématique le plaisir qu' il peut tirer de sa lecture.

C'est sur ce point que cette étude voudrait s'interroger, en abordant la question du lecteur du texte polémique d'un point de vue énonciatif et stylistique, et non spécifiquement didactique, tout en s'efforçant de mettre en lumière des problèmes qui ont une pertinence dans le champ didactique. Nous nous appuierons ici sur trois textes qui sont, sans nul doute, difficilement exploitables en classe, d'autant que les deux derniers posent d'évidents problèmes idéologiques et éthiques : "Avez-vous déjà giflé un mort??" "écrit par Aragon à l'occasion de la mort d'Anatole France, deux extraits du poème communiste "Front rouge ${ }^{3}$ ", du même auteur, et un extrait du pamphlet anti-communiste de Céline intitulé $\mathrm{Mea} \mathrm{culpa}^{4}$. Ces textes ont été choisis parce qu'ils possèdent des caractéristiques qui sont, à divers degrés, celles de tous les textes polémiques et, surtout, parce qu'ils font apparaitre, de manière évidente, les problèmes auxquels le lecteur du texte polémique peut être confronté, en tant que sujet, notamment lorsqu'il se trouve face à la violence d'un discours auquel il n'adhère pas nécessairement sur le plan idéologique, mais dont il peut néanmoins tirer un plaisir.

\section{Le piège du discours polémique}

Quelques éléments généraux de définition du polémique paraissent nécessaires avant toute analyse. Le polémique peut se définir comme un genre de discours, au sens de Tzvetan Todorov's, pouvant donner lieu à un discours de type littéraire, mais possédant des "parents» non littéraires. Le discours polémique, littéraire ou non, est caractérisé, sur le plan énonciatif, par une situation d'énonciation tripolaire : le discours se déploie dans le cadre de l'opposition entre deux adversaires, le polémiste et sa cible, opposition portée à la vue d'un tiers, le destinataire, qui joue le rôle de témoin et d'arbitre. Sur le plan pragmatique, le discours vise à gagner ce tiers à la cause de l'énonciateur, contre son adversaire, et il se définit ainsi, pour reprendre la terminologie jakobsonienne, par

2. L. Aragon, "Avez-vous déjà giflé un mort?» [1924], dans Tracts surréalistes et déclarations collectives, t. I, Paris, Éric Losfeld, I980, p. 24-25.

3. L. Aragon, «Front rouge» [1931], ibid., p. 459-465.

4. L.-F. Céline, Mea Culpa suivi de La Vie et l'Cuvre de Semmelweis, Paris, Denoël et Steele, I937, p. I7-I9.

5. T. Todorov, La Notion de littérature et autres essais, Paris, Seuil, 1987. Voir en particulier "L'origine des genres», p. 27-46. 
une dominante conative ${ }^{6}$. En raison de la structure énonciative tripolaire du discours polémique, cette fonction conative est double : elle se décompose en une fonction adversative, centrée sur la cible (le polémiste s'oppose à celle-ci et cherche, par exemple, à l'humilier) et une fonction conjonctive, centrée sur le destinataire (le polémiste cherche à gagner celui-ci à sa cause, par exemple, en s'efforçant de provoquer son indignation). Le discours polémique met ainsi en place deux relations énonciatives distinctes et simultanées, l'une entre le polémiste et sa cible, l'autre entre le polémiste et le destinataire. On est ici en présence de ce que Ducrot désigne comme l'éclatement de l'énonciation en une "multiplicité illocutoire ${ }^{\sharp}$ » : un même énoncé renvoie simultanément à deux relations énonciatives différentes. Celles-ci, et les fonctions qui les caractérisent, peuvent être ainsi représentées :

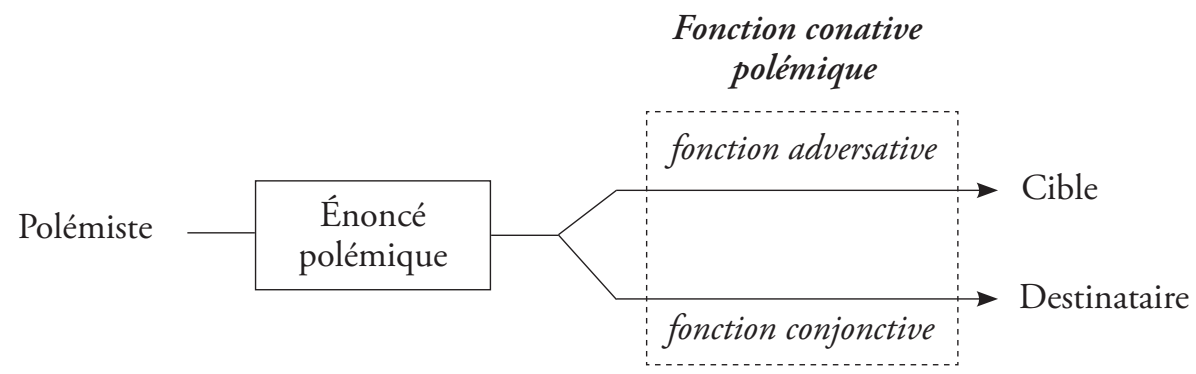

Ces deux relations peuvent être décrites comme distinctes, mais elles ne sont bien évidemment pas dissociables, le destinataire comme la cible «entendant» simultanément les deux lectures. Par exemple, le destinataire sourit d'une invective car il superpose à sa lecture celle que la cible, atteinte dans son amour-propre, est susceptible de faire. De son côté, la cible est d'autant

6. R. Jakobson, Essais de linguistique générale, Paris, Minuit, 1963. Voir en particulier le chap. II, "Linguistique et poétique».

7. O. Ducrot, Les Mots du discours, Paris, Minuit, 1980. Voir en particulier «Analyse de textes et linguistique de l'énonciation», p. 7-56. O. Ducrot décrit ainsi la «multiplicité illocutoire»: "Je précise que je ne parle pas ici des cas d'ambiguïté où deux sens différents font allusion, chacun, à un acte différent : ce qui m'intéresse, c'est la possibilité qu'une lecture unique d'un énoncé fasse éclater l'énonciation en une multiplicité illocutionnaire.» Prenant l'exemple de la phrase d'un ministre de l'Intérieur, "L'ordre sera maintenu coûte que coûte », il montre que l'énonciateur accomplit deux actes, un engagement et une menace, s'adressant à deux catégories distinctes de destinataires : "Pour décrire cette dualité, il est commode de dire que, si le ministre prend pour allocutaires l'ensemble des citoyens, en bloc, sans distinction, il se donne cependant deux catégories de destinataires. D'un côté, les bons citoyens, soucieux d'ordre et de tranquillité, auxquels il fait la promesse, et d'autre part les méchants, qui sont l'objet de la menace.» (p. 38-39) 
plus atteinte par l'invective qu'elle la sait publique et qu'elle a conscience que le destinataire sourit de son humiliation.

On s'intéressera ici au lecteur du texte polémique, en tant qu'il a une existence empirique et se distingue du destinataire qui, lui, n'a d'existence que textuelle, qui est construit dans le texte et par lui. Mais parler du lecteur implique aussi de s'interroger sur le destinataire : le lecteur est un sujet susceptible d'accepter de s'identifier au destinataire, d'occuper la place que le texte ménage à ce dernier, ou qui peut au contraire refuser de le faire. On limitera ici la réflexion à un cas de lecteur particulier : celui qui éprouve du plaisir à lire le texte polémique.

Le plaisir que le destinataire tire du discours polémique peut être décrit comme une forme de perversion : il est subordonné à la souffrance infligée à la cible, même si cette souffrance n'est que symbolique. Il s'agit, dans une certaine mesure au moins, d'un plaisir sadique : c'est de l'attaque de la cible et de son humiliation (au fondement de la fonction adversative du texte) que le destinataire tire son plaisir (que, dans sa fonction conjonctive, le texte s'efforce de générer). Le plaisir du lecteur n'existe quant à lui qu'à la condition qu'il accepte de jouer le rôle que le polémiste lui assigne, autrement dit qu'il accepte de s'identifier au destinataire. Le discours enferme ainsi son lecteur dans un rôle et lui tend un "piège», qui peut se formuler ainsi : soit le lecteur adhère à la cause du polémiste et il est placé dans la position supérieure de celui qui, aux côtés du polémiste, méprise la cible; soit il fait corps avec la cible et il encourt le mépris du polémiste, il subit lui aussi la violence du discours, se trouvant dans l'impossibilité de tirer un quelconque plaisir de la lecture.

Pour illustrer le piège que le texte polémique tend à son lecteur ainsi que la violence face à laquelle il le place, nous nous appuierons sur «Avez-vous déjà giflé un mort?", qu'Aragon signe à l'occasion de la mort d'Anatole France et qui paraît dans l'opuscule Un cadavre ${ }^{8}$. Voici ce texte, dans son intégralité :

La colère me prend si, par quelque lassitude machinale, je consulte parfois les journaux des hommes. C'est qu'en eux se manifeste un peu de cette pensée commune, autour de laquelle, vaille que vaille, un beau jour, ils tombent d'accord. Leur existence est fondée sur une croyance en cet accord, c'est là tout ce qu'ils exaltent, et il faut pour qu'un homme recueille encore leurs suffrages, pour qu'aussi un homme recueille les suffrages des derniers des hommes, qu'il soit une figure évidente, une matérialisation de cette croyance.

8. Outre le texte d'Aragon, paraissent dans ce volume des textes de Ph. Soupault («L'erreur»), de P. Éluard («Un vieillard comme les autres»), de P. Drieu La Rochelle («Ne nous la faites pas à l'oseille»), de J. Delteil ("Anatole France ou la médiocrité dorée») et de A. Breton ("Refus d'inhumer»). 
Les conseils municipaux de localités à mes yeux indistinctes s'émeuvent aujourd'hui d'une mort, posent au fronton de leurs écoles des plaques où se lit un nom. Cela devrait suffire à dépeindre celui qui vient de disparaître, car l'on n'imagine pas Baudelaire, par exemple, ou tout autre qui se soit tenu à cet extrême de l'esprit qui seul défie la mort, Baudelaire célébré par la presse et ses contemporains comme un vulgaire Anatole France. Qu'avait-il, ce dernier, qui réussisse à émouvoir tous ceux qui sont la négation même de l'émotion et de la grandeur? Un style précaire, et que tout le monde se croit autorisé à juger par le vou même de son possesseur; un langage universellement vanté quand le langage pourtant n'existe qu'au-delà, en dehors des appréciations vulgaires. Il écrivait bien mal, je vous le jure, l'homme de l'ironie et du bon sens, le piètre escompteur de la peur du ridicule. Et c'est encore très bien que de bien écrire, que d'écrire, auprès de ce qui mérite un seul regard. Tout le médiocre de l'homme, le limité, le peureux, le conciliateur à tout prix, la spéculation à la manque, la complaisance dans la défaite, le genre satisfait, prudhomme, niais, roseau pensant, se retrouvent, les mains frottées, dans ce Bergeret dont on me fera vainement valoir la douceur. Merci, je n'irai pas finir sous ce climat facile une vie qui ne se soucie pas des excuses et du qu'en dira-t-on.

Je tiens tout admirateur d'Anatole France pour un être dégradé. Il me plaît que le littérateur que saluent à la fois aujourd'hui le tapir Maurras et Moscou la gâteuse, et par une incroyable duperie Paul Painlevé lui-même, ait écrit pour battre monnaie d'un instinct tout abject, la plus déshonorante des préfaces à un conte de Sade, lequel a passé sa vie en prison pour recevoir à la fin le coup de pied de cet âne officiel. Ce qui vous flatte en lui, ce qui le rend sacré, qu'on me laisse la paix, ce n'est même pas le talent, si discutable, mais la bassesse, qui permet à la première gouape venue de s'écrier : «Comment n’y avais-je pas pensé plus tôt!» Exécrable histrion de l'esprit, fallait-il qu'il répondît vraiment à l'ignominie française pour que ce peuple obscur fût à ce point heureux de lui avoir prêté son nom! Balbutiez donc à votre aise sur cette chose pourrissante, pour ce ver qu'à son tour les vers vont posséder, râclures de l'humanité, gens de partout, boutiquiers et bavards, domestiques d'état, domestiques du ventre, individus vautrés dans la crasse et l'argent, vous tous, qui venez de perdre un si bon serviteur de la compromission souveraine, déesse de vos foyers et de vos gentils bonheurs.

Je me tiens aujourd'hui au centre de cette moisissure, Paris, où le soleil est pâle, où le vent confie aux cheminées une épouvante et sa langueur. Autour de moi, se fait le remuement immonde et misérable, le train de l'univers où toute grandeur est devenue l'objet de la dérision. L'haleine de mon interlocuteur est empoisonnée par l'ignorance. En France, à ce qu'on dit, tout finit en chansons. Que donc celui qui vient de crever au cour de la béatitude générale, s’en aille à son tour en fumée! Il reste peu de choses d'un homme : il est encore révoltant d'imaginer de celui-ci, que de toute façon il a été. Certains jours j'ai rêvé d'une gomme à effacer l'immondice humaine'.

Le troisième paragraphe formule clairement le piège dans lequel le texte s'efforce de prendre le lecteur, qui n'a ici que deux attitudes possibles : soit il

9. L. Aragon, "Avez-vous déjà giflé un mort?», dans Tracts surréalistes et déclarations collectives, ouvr. cité, p. 24-25. 
méprise Anatole France, soit il encourt le mépris d'Aragon. Le piège construit par le discours polémique n'est pas toujours aussi visible que dans ce texte, mais il fonde toujours l'efficacité du discours, qui mise sur le fait que, si le lecteur veut tirer du plaisir de sa lecture, il doit adhérer à la cause du polémiste. Dans le cas contraire, sa position se transforme : il n'est plus dans la position du tiers témoin de l'agression mais se trouve assimilé à la cible elle-même et donc mis dans la position de celui qui est agressé, c'est-à-dire de celui pour qui le texte est destiné à générer du déplaisir, et non du plaisir. Si le lecteur veut rire avec le polémiste, il est obligé de se ranger, au moins le temps de sa lecture, de son côté.

\section{Le lecteur face à la violence textuelle}

Autre caractéristique du discours polémique : il met toujours son lecteur face à une violence discursive. La relation mise en place entre le polémiste et la cible transgresse, à des niveaux variés, les codes de la communication policée. Dans le texte d'Aragon, la transgression la plus manifeste est, bien entendu, celle qui consiste à attaquer un mort encore «tiède», si l'on peut dire, les détracteurs d'une personne se devant d'observer une période de silence à sa mort. La violence provocatrice de l'image utilisée dans le titre traduit cette transgression en termes physiques.

D’une manière générale, la transgression des codes de la communication policée s'opère dans le texte polémique par l'intermédiaire de deux formes de violence symbolique, exercées par le polémiste à l'égard de sa cible : l'injure et la mise à mort. L'injure, fréquente dans le discours polémique, n'est certes qu'un substitut symbolique de la violence physique, comme Freud le rappelle dans Le Mot d'esprit et sa relation à l'inconscient :

Les impulsions hostiles qui nous poussent contre nos semblables sont soumises, depuis notre enfance individuelle comme depuis celle de la civilisation humaine, aux mêmes limitations, au même refoulement progressif que nos aspirations sexuelles. [...] Interdite par la loi, l'hostilité qui se traduit par des actes de violence a été relayée par l'invective, laquelle se sert de mots [...]. Doués de fortes prédispositions à l'hostilité alors que nous sommes encore enfants, l'éducation individuelle qui nous rend plus civilisés nous enseigne plus tard que c'est manquer de dignité que d'avoir recours aux insultes $[\ldots]^{\text {to }}$.

Mais quelque symbolique qu'elle soit, la violence de l'injure reste transgressive, puisqu'elle est normalement exclue de la communication policée.

Io. S. Freud, Le Mot d'esprit et sa relation à l'inconscient, Paris, Gallimard, 2006, p. 197-198. 
Dans le texte d'Aragon, la relation entre la violence physique à laquelle le titre réfère (la gifle) et sa traduction verbale (l'injure) est claire. Les syntagmes qui permettent de désigner la cible mettent en œuvre cette violence verbale. Ainsi, dans le titre, le polémiste refuse de nommer celui auquel il s'attaque, recourant à une expression indéfinie ("un mort»), qui le réduit à l'anonymat, qui en fait une simple occurrence d'un ensemble de référents. Ce n'est que dans le deuxième paragraphe que la cible est nommée, et cette première apparition du nom propre est intéressante : "[...] on n’imagine pas Baudelaire, par exemple, ou tout autre qui se soit tenu à cet extrême de l'esprit qui seul défie la mort, Baudelaire célébré par la presse et ses contemporains comme un vulgaire Anatole France». Le polémiste ne sort la cible de l'anonymat que pour mieux l'y replonger, par l'intermédiaire de l'antonomase, faisant du nom propre un nom commun, déterminé par un indéfini, susceptible de désigner un groupe de référents ayant des caractéristiques semblables, qualifié qui plus est par l'adjectif «vulgaire». L'opposition entre ce traitement stylistique du nom propre et celui du nom de Baudelaire, répété et traité comme un véritable nom propre, est bien évidemment très révélatrice. À la fin du troisième paragraphe, on peut commenter les syntagmes "cette chose pourrissante» et "ce ver qu'à son tour les vers vont posséder ", déterminés par des démonstratifs à valeur manifestement dépréciative, qui désignent la cible par l'intermédiaire de termes qui la font sortir de la catégorie de l'humain, par réification et animalisation. On relève la violence de l'évocation de la disparition physique, par l'étrange verbe "posséder", qui génère des images dérangeantes d'une espèce d'accouplement monstrueux.

D'une manière générale, et sans qu'il soit nécessaire de commenter tous les syntagmes qui désignent la cible, on peut observer une tendance marquée dans le texte du passage de la détermination indéfinie (article indéfini, notamment dans le titre et devant le nom propre) à la détermination définie (article défini puis démonstratif). La violence du polémiste consiste d'abord à dénier à la cible son individualité, à la réduire à l'anonymat et ainsi à l'affaiblir, pour ensuite la désigner, par les démonstratifs, au mépris du destinataire. Il organise, sur le plan symbolique, une forme de lynchage de son adversaire. La violence symbolique qui se déploie dans le texte peut être ainsi décrite par analogie avec un combat dans lequel le polémiste désarme son adversaire pour ensuite l'achever.

Le polémiste établit avec la cible un rapport de force violent répondant au désir de l'éliminer en tant qu'adversaire, de l'annihiler en tant que locuteur, de la réduire au silence. L'horizon du discours polémique est ainsi toujours la mise à mort de l'autre. Shoshana Felman le souligne : "Quel que soit le sujet de la polémique, le discours polémique est toujours, à un certain niveau, un discours sur la mort, de la mort, un discours sur l'acte de tuer. L'enjeu de la 
polémique, si symbolique soit-il, est le meurtre de l'adversaire ${ }^{\mathrm{II}}$.» Cette mise à mort est généralement métaphorique et symbolique, mais elle est parfois explicitement évoquée. Dans le texte d'Aragon, la situation est un peu particulière, puisque la cible est déjà morte, mais le texte la met d'une certaine manière à mort une seconde fois à la fin :

En France, à ce qu'on dit, tout finit en chansons. Que donc celui qui vient de crever au cour de la béatitude générale, s'en aille à son tour en fumée! Il reste peu de choses d'un homme : il est encore révoltant d'imaginer de celui-ci, que de toute façon il a été. Certains jours j'ai rêvé d'une gomme à effacer l'immondice humaine.

La violence est ainsi au cœur du discours polémique. Mais, pour que celui-ci reste efficace, cette violence est soumise à au moins deux règles. D'abord, celle qu'on nommera règle de la juste mesure: le discours doit agresser la cible sans s'aliéner le lecteur. Le polémiste doit prendre en compte le seuil au-delà duquel la violence serait inacceptable pour le lecteur et où, par conséquent, il se rangerait aux côtés de la cible et/ou cesserait de lire. Ensuite, la règle qu'on appellera, en empruntant l'expression à Freud, la règle du "gain de plaisir»: le discours doit non seulement ne pas s'aliéner le lecteur par une violence excessive, mais il doit également le séduire. Le polémiste est dans une situation similaire à celle de l'un des types décrits par Freud dans Le Mot d'esprit et sa relation à l'inconscient: l'agresseur du mot d'esprit à tendance hostile. Le mot d'esprit, comme le texte polémique, "va soudoyer le lecteur grâce au gain de plaisir qu'il lui procure, obtenant de lui qu'il prenne notre parti sans procéder à un examen rigoureux ${ }^{12} »$.

\section{Le gain de plaisir}

Ce "gain de plaisir », comme d'ailleurs la «juste mesure», est bien sûr variable d'un sujet lecteur à l'autre, mais on peut observer la manière dont le texte vise à le provoquer, identifier des réseaux de procédés convergeant vers la production de cet effet. Pour cela, on se fondera sur deux textes qui posent des

II. S. Felman, «Le discours polémique (Propositions préliminaires pour une théorie de la polémique)", Cahiers de l'Association internationale des études françaises, $\mathrm{n}^{\circ}$ 31, mai 1979, p. 187. I2. S. Freud, Le Mot d'esprit et sa relation à l'inconscient, ouvr. cité, p. 199. On retrouve, dans la description que propose Freud, la structure tripolaire caractéristique du polémique : «À partir du moment où nous avons dû renoncer à exprimer notre hostilité par des actes [...], nous avons commencé $[\ldots]$ à développer une nouvelle technique, celle de l'outrage, qui a pour but de recruter cette tierce personne dans la lutte contre notre ennemi. En rendant l'ennemi petit, bas, méprisable, comique, nous réussissons par un biais à jouir de l'avoir dominé, jouissance dont la tierce personne [...] nous donne témoignage par son rire.» (p. 198-I99) 
problèmes de lecture particuliers, la violence transgressive qu'ils mettent en œuvre étant très forte : un extrait de Mea Culpa, pamphlet anti-communiste de Céline, daté de 1937, et deux extraits de «Front rouge», poème communiste d'Aragon appelant à la violence prolétarienne, publié en 193i. Notre objectif sera d'observer ces deux textes du point de vue de la relation entre violence et gain de plaisir.

Céline, dans Mea Culpa, prend pour cible le communisme et, dans l'extrait choisi, convoque à ses côtés la religion, dont il montre la supériorité. En réalité, la verve du polémiste s'exerce aussi à l'égard de l'adjuvant qu'il feint de se donner.

Le Communisme matérialiste, c'est la Matière avant tout et quand il s'agit de matière c'est jamais le meilleur qui triomphe, c'est toujours le plus cynique, le plus rusé, le plus brutal. Regardez donc dans cette URSS comme le pèze s'est vite requinqué! Comme l'argent a retrouvé tout de suite toute sa tyrannie! et au cube encore! Pourvu qu'on le flatte, le Popu prend tout! avale tout! Il est devenu là-bas hideux de prétention, de suffisance, à mesure qu'on le faisait descendre plus profond dans la mouscaille, qu'on l'isolait davantage! C'est ça l'effrayant phénomène. Et plus il se rend malheureux, plus il devient crâneur! Depuis la fin des croyances, les chefs exaltent tous ses défauts, tous ses sadismes, et le tiennent plus que par ses vices : la vanité, l'ambition, la guerre, la Mort en un mot. Le truc est joliment précieux! Ils ont repris tout ça au décuple! On le fait crever par la misère, par son amour-propre aussi! Vanité d'abord! La prétention tue comme le reste! Mieux que le reste!

La supériorité pratique des grandes religions chrétiennes, c'est qu'elles doraient pas la pilule. Elles essayaient pas d'étourdir, elles cherchaient pas l'électeur, elles sentaient pas le besoin de plaire, elles tortillaient pas du panier. Elles saisissaient l'Homme au berceau et lui cassaient le morceau d'autor. Elles le rencardaient sans ambages : «Toi petit putricule informe, tu seras jamais qu'une ordure... De naissance tu n'es qu'une merde... Est-ce que tu m'entends?... C'est l'évidence même, c'est le principe de tout! Cependant, peut-être... peut-être... en y regardant de tout près... que t'as encore une petite chance de te faire un peu pardonner d'être comme ça tellement immonde, excrémentiel, incroyable... C'est de faire bonne mine à toutes les peines, épreuves, misères et tortures de ta brève ou longue existence. Dans la parfaite humilité... La vie, vache, n'est qu'une âpre épreuve! T'essouffle pas! Cherche pas midi à quatorze heures! Sauve ton âme, c'est déjà joli ! Peut-être quà la fin du calvaire, si t'es extrêmement régulier, un héros "de fermer ta gueule", tu claboteras dans les principes... Mais c'est pas certain... un petit poil moins putride à la crevaison qu'en naissant... et quand tu verseras dans la nuit plus respirable qu'à l'aurore... Mais te monte pas la bourriche! C'est bien tout! Fais gaffe! Spécule pas sur les grandes choses! Pour un étron c'est le maximum!...»

Ça! c’était sérieusement causé! Par des vrais Pères de l’Église! Qui connaissaient leur ustensile! qui se miroitaient pas d'illusions ${ }^{13}$ !

I3. L.-F. Céline, Mea Culpa, ouvr. cité, p. I7-I9. 
La violence transgressive du texte, et en particulier du deuxième paragraphe de l'extrait, tient à ce qu'il transpose le discours chrétien dans une langue argotique, parfois grossière, et marquée par le scatologique qui désigne, métaphoriquement, la misère de la condition humaine. Le texte est fortement blasphématoire, il joue à la limite de l'acceptable et, surtout, à la limite de l'efficace : le risque est ici que le lecteur abandonne la lecture ou se range du côté de la cible, considérant que le polémiste a enfreint la règle de la «juste mesure».

Mais un certain nombre de procédés visent à faire accepter au lecteur la violence transgressive en échange d'un gain de plaisir. Dans les phrases qui ouvrent le deuxième paragraphe, d'abord, et qui procèdent par paraphrase de l'expression initiale "dorer la pilule", le texte mise sur le plaisir qu'est susceptible de générer la profusion des synonymes, une espèce de surabondance de la langue argotique. Par ailleurs, ces phrases annoncent le discours rapporté tout en le retardant, par leur accumulation, générant ainsi un désir du lecteur, que le texte vient ensuite combler, provoquant un gain de plaisir. Dans la séquence en discours rapporté, ensuite, on note qu'il n'y a pas un seul mot appartenant au lexique religieux : le discours religieux est traduit, transposé dans une langue vulgaire. Le gain de plaisir, sur lequel le texte mise pour compenser ou contrebalancer la violence blasphématoire, est ici fondé sur la reconnaissance du discours religieux : il s'agit du plaisir, pour le lecteur, d'exercer, même à peu de frais, ses capacités interprétatives. Sans créer nécessairement une véritable connivence avec le polémiste, le texte donne au lecteur le plaisir qu'il tire toujours d'un discours qui mise sur sa capacité à reconnaitre une allusion, un implicite. Le choix de la forme directe du discours rapporté est également intéressant : fondée sur une opération de citation, cette forme suppose une fidélité sur le plan de la matérialité du message rapporté, qui relève évidemment ici de l'impossible : le gain de plaisir que le texte s'efforce de générer est lié au comique qui naît de la tension entre le signifié religieux (le discours sur la misère humaine et la grâce divine, qui appellerait un registre élevé) et le signifiant (un discours qui relève du bas et du trivial). Si l'on s'amuse à réécrire la séquence en discours indirect $\mathrm{t}^{14}$, le texte perd toute son efficacité : le niveau de langue et le style (la métaphore scatologique en particulier) seraient attribués au locuteur premier, le polémiste, et non plus, comme c'est le cas ici, au locuteur second, la religion.

Le degré de transgression de ce texte est fort, et des procédés visant le gain de plaisir du lecteur sont mis en place, afin de rendre le texte lisible. Mais

I4. Elles le rencardaient sans ambages, lui lançant que lui, petit putricule informe, il serait jamais qu'une ordure... que de naissance il n'était qu'une merde... Est-ce qu'il l'entendait?... C'était l'évidence même, c'était le principe de tout... etc. 
évidemment, ce gain de plaisir est problématique : le lecteur peut refuser de l'éprouver précisément car le prix en est d'accepter d'être le témoin, et peutêtre d'une certaine manière la caution ou le complice, d'une transgression très forte.

Dans «Front rouge», Aragon en appelle à la violence prolétarienne, incitant au meurtre des «flics» et de ceux qu'il appelle les «ours de la social-démocratie». Le numéro de Littérature de la révolution mondiale ${ }^{15}$ dans lequel le texte paraît est interdit, saisi par la police française et Aragon est inculpé de "provocation au meurtre dans un but de propagande anarchiste ${ }^{16}{ }_{\text {» }}$.

Dans ce premier extrait, qui frappe par sa violence, l'auteur formule l'appel au meurtre de manière très explicite et désigne nommément certaines de ses cibles :

Pliez les réverbères comme des fétus de paille

faites valser les kiosques les bancs les fontaines Wallace

Descendez les flics

Camarades

Descendez les flics

$[\ldots]$

Prolétariat connais ta force

Connais ta force et déchaîne-la

Il prépare son jour il attend son heure

sa minute la seconde

où le coup porté sera mortel

et la balle à ce point sûre que tous les médecins social-fascistes

penchés sur le corps de la victime

auront beau promener leurs doigts chercheurs sur la chemise de dentelles

ausculter avec des appareils de précision son cœur déjà pourrissant

ils ne trouveront pas le remède habituel

et tomberont aux mains des émeutiers qui les colleront au mur

Feu sur Léon Blum

Feu sur Boncour Frossart Déat

Feu sur les ours savants de la social-démocratie

Feu Feu j'entends passer

la mort qui se jette sur Garchery Feu vous dis-je

Sous la conduite du Parti communiste ${ }^{17}$

I5. Littérature de la Révolution mondiale, $\mathrm{n}^{\circ}$ I, juillet I93I. Édité en plusieurs langues, à Moscou, Littérature de la Révolution mondiale est l'organe central de l'Union internationale des écrivains révolutionnaires.

I6. Sur «Front rouge» et, plus largement, sur certains appels au meurtre formulés par Aragon et Breton, voir P. Roux, «Appels au meurtre surréalistes : Breton, Aragon », dans F. Quinche et A. Rodriguez (dir.), Quelle éthique pour la littérature? Pratiques et déontologies, Genève, Labor et Fides, 2007, p. 57-78.

I7. L. Aragon, «Front rouge» [1931], dans Tracts surréalistes et déclarations collectives, ouvr. cité, p. 46I. 
Même si l'on admet qu'on est dans le cadre d'une violence symbolique hypothèse que la justice n'a, semble-t-il, pas retenue - la dimension transgressive domine et le texte ne semble pas organisé pour générer un gain de plaisir du lecteur, si ce n'est peut-être le plaisir de la transgression elle-même. Les choses sont tout à fait différentes dans l'extrait suivant :

Dans les plâtras croulants

parmi les fleurs fanées des décorations anciennes

les derniers napperons et les dernières étagères

soulignent la survie étrange des bibelots

Le ver de la bourgeoisie

essaye en vain de joindre ses tronçons épars

Ici convulsivement agonise une classe

les souvenirs de famille s'en vont en lambeaux

Mettez votre talon sur ces vipères qui se réveillent

Secouez ces maisons que les petites cuillères

en tombent avec les punaises la poussière des vieillards

qu'il est doux qu'il est doux le gémissement qui sort des ruines

J'assiste à l'écrasement d'un monde hors d'usage

J'assiste avec enivrement au pilonnage des bourgeois

Y a-t-il jamais eu plus belle chasse que l'on donne

à cette vermine qui se tapit dans tous les recoins des villes

Je chante la domination violente du Prolétariat sur la bourgeoisie

pour l'anéantissement de cette bourgeoisie

pour l'anéantissement total de cette bourgeoisie

Le plus beau monument qu'on puisse élever sur une place

la plus surprenante de toutes les statues

la colonne la plus audacieuse et la plus fine

l'arche qui se compare au prisme même de la pluie

ne valent pas l'amas splendide et chaotique

Essayez pour voir

Qu'on produit aisément avec une église et de la dynamite

La pioche fait une trouée au cœur des docilités anciennes

Les écroulements sont des chansons où tournent des soleils

Hommes et murs d'autrefois tombent frappés de la même foudre

L'éclat des fusillades ajoute au paysage

une gaieté jusqu'alors inconnue

Ce sont des ingénieurs des médecins qu'on exécute

Mort à ceux qui mettent en danger les conquêtes d'Octobre

Mort aux saboteurs du Plan Quinquennal ${ }^{18}$

Le propos et sa violence sont identiques. Le meurtre n'est cependant plus seulement présenté comme un acte politique nécessaire mais comme une source de joie et de plaisir esthétique, ce qui, en un sens, augmente encore la force transgressive de l'appel proféré. Et le texte est organisé pour générer un gain

18. Ibid., p. 462. 
de plaisir du lecteur, qui fait pendant au plaisir qu' exprime le poète lui-même. Énonciateur et destinataire sont ainsi appelés par le texte à communier dans un plaisir problématique, qui plonge ses racines dans la violence du meurtre. Pour illustrer ce point, on se contentera ici de commenter la troisième strophe citée («Le plus beau monument qu’on puisse élever sur une place $[\ldots] »)$.

L'information principale (l'appel à faire sauter les églises) est située dans le dernier vers, et la strophe est tout entière organisée pour la retarder et ainsi générer un désir du lecteur : l'accumulation des groupes nominaux qui constituent le sujet, les superlatifs, un lexique hyperbolique, la proposition incidente, finalement, qui retarde encore l'information principale en interpellant le lecteur. Le dernier vers, attendu, génère un plaisir, en même temps qu'il profere l'appel à la violence. Autrement dit, Aragon parvient, par la syntaxe, à faire qu'advienne le gain de plaisir du lecteur au moment-même où culmine la violence transgressive de l'énoncé.

Ce dernier vers, qui constitue une chute contrastive, avec un lexique qui n'est plus hyperbolique ni poétique, génère par ailleurs un effet de rétrolecture. Il incite à relire la strophe comme une métaphore de l'explosion : "l'amas splendide et chaotique" évoque les ruines résultant de l'explosion et la série des quatre expressions qui constituent le sujet peuvent être relus a posteriori comme des métaphores du moment même de l'explosion. Ce qui est ici remarquable, c'est que l'effet de rétrolecture génère non seulement un plaisir du lecteur, lié, pour aller vite, à la surprise, mais qu’il génère en plus un désir de relire la strophe.

Ces deux effets du texte (mise en suspens et rétrolecture) sont d'autant plus puissants qu'ils se déploient dans l'espace resserré de la phrase. L'information principale, c'est-à-dire celle qu'attend le lecteur et celle qui lui permet de saisir la véritable signification de la séquence, est rejetée à la fin, dans des constituants accessoires sur le plan syntaxique : la relative finale ("qu'on produit aisément avec une église et de la dynamite») et la proposition incidente ("essayez pour voir») qui équivalent, sur le plan sémantique, à une injonction du type : «Faites sauter les églises». L'information principale est ainsi reléguée, pourrait-on dire, dans des constituants de niveau hiérarchique inférieur (subordonnée ou incidente). Le plaisir de la lecture est lié en partie à l'identification de cette inversion hiérarchique qui met en tension la syntaxe de la phrase (qui établit une hiérarchie entre constituants) et la signification de la séquence (qui impose une autre hiérarchie entre les éléments). Le gain de plaisir du lecteur tient ici à l'exercice de ses capacités interprétatives, qui lui permettent d'identifier et d'interpréter cette tension entre l'ordre de la syntaxe et l'ordre de la signification.

Il suffit ici de réorganiser autrement l'information dans la phrase, en supprimant l'effet de mise en suspens et en mettant en harmonie syntaxe et 
signification, pour constater que tout le gain de plaisir est perdu : «On produit aisément avec une église et de la dynamite - essayez pour voir - un amas splendide et chaotique qui surpasse le plus beau monument qu'on puisse élever sur une place..."

Le plaisir de la lecture du texte polémique est toujours un plaisir problématique, que le lecteur est libre d'accepter ou de refuser. Les deux textes présentés posent un problème idéologique spécifique, dans la mesure où ils correspondent à des positions inacceptables pour la majorité des lecteurs. Et ils montrent par là même que le gain de plaisir est en partie indépendant du contenu idéologique du texte, au point qu'il peut permettre au lecteur de surmonter, temporairement, l'incompatibilité de ses valeurs avec celles du texte. On peut ne pas adhérer idéologiquement à l'appel à la terreur lancé par Aragon, et pourtant tirer de la lecture du texte un plaisir. Dire que ce passage de «Front rouge» est efficace, ce n'est pas dire qu'il donne nécessairement au lecteur le désir de passer à l'acte, mais que celui-ci peut éprouver un gain de plaisir malgré le fait qu'il n'adhère pas idéologiquement à la position prise par le polémiste.

Le texte polémique pose ainsi à tout lecteur un problème éthique : vais-je accepter, si l'on peut dire, cet "argent sale» que me propose le polémiste, ce gain de plaisir à l'origine duquel et derrière lequel se trouve une violence qui transgresse les lois de la société civilisée? Et dans un contexte didactique, il pose des problèmes encore plus complexes : étant donnée la position très particulière du lecteur du texte polémique, peut-on faire étudier, et peut-être faire aimer le texte polémique? N'y a-t-il pas un danger à mettre des sujets qui n'ont peut-être pas atteint leur pleine maturité de lecteurs face à cette violence?

Sans prétendre apporter une réponse à cette question, on peut, en guise de conclusion, proposer quelques pistes. Si l'on considère qu'en situation didactique, le sujet lecteur ne saurait être mis dans une telle position, alors il faudrait exclure tous les textes polémiques. $\mathrm{Si}$, au contraire, on considère que, malgré ces spécificités, le texte polémique est exploitable, alors il faut, semble$\mathrm{t}$-il, travailler à partir du plaisir problématique que chaque sujet tire (ou ne tire pas, ou encore refuse de tirer) de la lecture. La question de l'expérience de lecture doit être centrale, et elle doit être déconstruite, afin de mettre à distance les effets d'adhésion que le texte peut produire à la première lecture. Le texte polémique est peut-être, si on l'aborde ainsi, un support privilégié de l'apprentissage de la lecture critique.

Autre question : si l'on fait étudier des textes polémiques, faut-il ne choisir, comme c'est semble-t-il toujours le cas, que des textes qui défendent des valeurs sur lesquelles il y a consensus, au moins de l'institution et de l'enseignant? Cette question en recoupe une qui ne se pose pas seulement à l'enseignant, 
mais aussi au chercheur : peut-on étudier la langue d'un auteur qui défend des positions inacceptables? Reformulé de manière un peu simpliste : a-t-on le droit de dire d'un auteur qui pense mal qu'il écrit bien, ou en tout cas que l'étude de sa langue présente un intérêt? C'est bien là tout le débat qui a lieu actuellement autour d'auteurs comme Céline ou Drieu La Rochelle.

Évidemment, la solution la plus simple, et peut-être la plus sage, surtout en contexte didactique, est de ne choisir que des textes dont les valeurs sont consensuelles. Mais peut-être les textes dont les valeurs se présentent comme inacceptables peuvent-ils avoir un intérêt, précisément parce qu'ils font éprouver au sujet lecteur un "gain de plaisir " qui va à l'encontre de ses valeurs. Lire des textes polémiques auxquels on n'adhère pas, déconstruire sa lecture, c'est aussi apprendre à distinguer plaisir du texte et idéologie. C'est apprendre à mettre à distance le plaisir, à savoir l'évaluer à sa juste valeur, comprendre son fonctionnement, ne pas le confondre avec l'adhésion intellectuelle et ainsi, peut-être, construire sa liberté de lecteur. 